\title{
Description of a human direct calorimeter, with a note on the energy cost of clerical work
}

\author{
BY JOAN D. WEBSTER, GILLIAN WELSH, P. PACY \\ AND J. S. GARROW* \\ Nutrition Research Group, Clinical Research Centre, Watford Road, Harrow HAl $3 U J$
}

(Received 26 November 1984 - Accepted 24 July 1985)

\begin{abstract}
1. A heat-sink calorimeter, suitable for the measurement of energy expenditure in human subjects over periods up to $26 \mathrm{~h}$, is described.

2. The performance of the calorimeter is illustrated by a study of four normal subjects at rest or performing clerical work for a period of $7.5 \mathrm{~h}$. Each condition was measured in duplicate in each subject. On the resting days the subjects were recumbent, and on the working days they were seated throughout the measurement period. Heart rate was monitored by infra-red telemetry and physical activity by an ultrasound movement detector. Urinary cortisol excretion was also measured as an indicator of stress.

3. In each subject the mean heat loss on working days was higher than that on resting days: the increase ranged from $5.1 \%$ to $16.7 \%$, with a mean value of $10.0 \%(P=0.015)$. There was no significant difference between resting and working days in heart rate, physical activity or urinary cortisol excretion.

4. The present study confirms that tiring clerical work has very little effect on $24 \mathrm{~h}$ energy expenditure.
\end{abstract}

With a properly designed calorimeter it is possible to measure the heat loss of human subjects over a period of $24 \mathrm{~h}$ with an accuracy better than $2 \%$ (Blaza \& Garrow, 1983), but the measurements always relate to a subject in a calorimeter, and it is difficult to estimate the effect of confinement in a calorimeter chamber on the energy expenditure of the subject.

It has been suggested that anxiety may cause a significant increase in metabolic rate (Corey, 1948; Udalov \& Sibunev, 1964; Tremoliere et al. 1973). Blaza \& Garrow (1980) induced stress experimentally in normal volunteers by mental arithmetic and electric shocks, and noted an increase of about $4 \%$ in metabolic rate. Benedict \& Benedict (1933) failed to show any difference in metabolic rate when mathematicians were, or were not, solving mathematical problems: they concluded that there was no measurable energy cost in mental work. Landis (1925) subjected himself and colleagues to severe discomfort and sleep deprivation, but obtained equivocal effects on metabolic rate.

The present study was designed to investigate the effect of a more usual type of stress on metabolic rate. Measurements were made by direct calorimetry on normal subjects on two occasions relaxing on a bed in a direct calorimeter, and on another two occasions while performing quite arduous clerical work which involved prolonged concentration, but negligible physical activity.

\section{METHODS}

The subjects were all members of staff in the Nutrition Research Group. Their physical characteristics are given in Table 1.

The direct calorimeter is illustrated in Fig. 1. The room in which it was situated was maintained at a constant temperature by the air-conditioning unit (AC): for these experiments the temperature was $27^{\circ}$. The calorimeter consisted of a chamber with internal dimensions $2 \mathrm{~m}$ wide, $1.7 \mathrm{~m}$ deep and $2 \mathrm{~m}$ high, made from expanded polystyrene $200 \mathrm{~mm}$ 
Table 1. Characteristics of subjects

\begin{tabular}{cccccc}
\hline Subject & Sex & $\begin{array}{c}\text { Age } \\
\text { (years) }\end{array}$ & $\begin{array}{c}\text { Wt }(\mathrm{W}) \\
(\mathrm{kg})\end{array}$ & $\begin{array}{c}\text { Height }(\mathrm{H}) \\
(\mathrm{m})\end{array}$ & $\mathrm{W} / \mathrm{H}^{2}$ \\
\hline G.W. & $q$ & 21 & 54.4 & 1.60 & 21.3 \\
J.W. & + & 29 & 74.9 & 1.75 & 24.5 \\
J.G. & 0 & 55 & 80.8 & 1.82 & 24.4 \\
P.P. & 0 & 32 & 55.3 & 1.71 & 18.9 \\
\hline
\end{tabular}

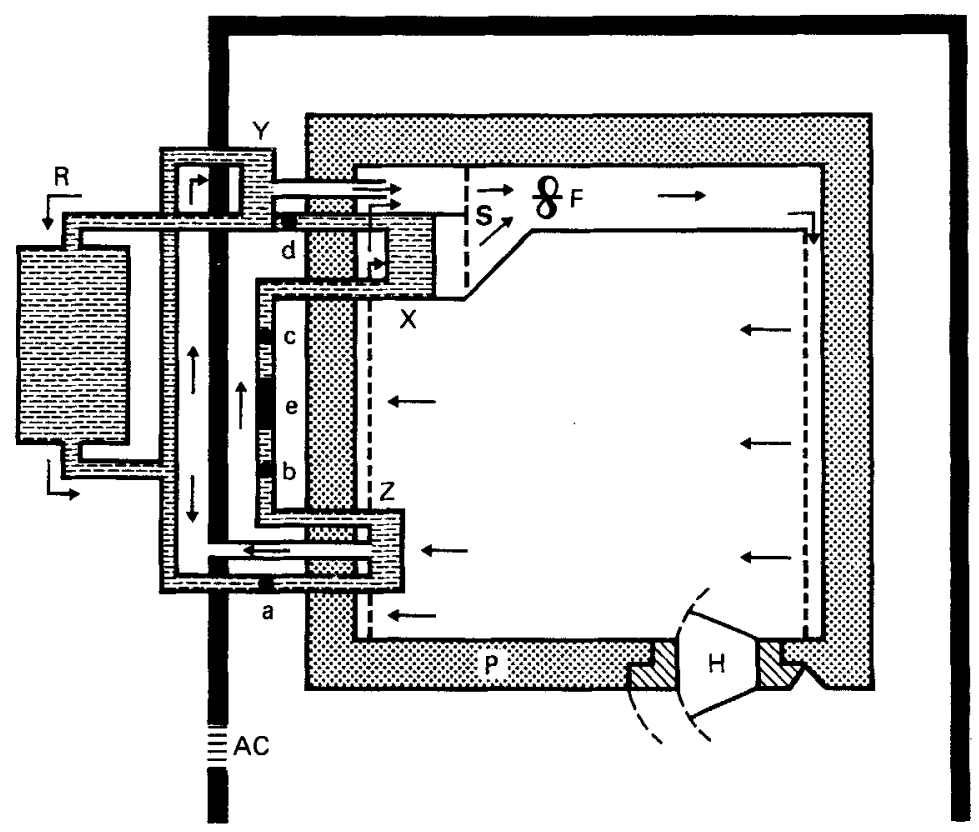

Fig. 1. Diagram of the direct calorimeter constructed by the Division of Bioengineering at the Clinical Research Centre. AC, air-conditioning unit; P, polystyrene; H. hatch; F, fan; S, shutter, X, Y and Z, heat exchangers; $R$, reservoir; e, reference heater; $a, b, c$ and d, thermistors.

thick (P), covered on both internal and external faces with aluminium sheet. The space which could be occupied by the subject was reduced in width by $100 \mathrm{~mm}$ by perforated hardboard false walls at either end, and in height by $20 \mathrm{~mm}$ by a wooden floor. A large panoramic photograph covered one internal wall. There was a door with a pass-through hatch $(\mathrm{H})$ which had internal and external doors of clear plastic. The internal door of the hatch had a rubber sleeve which would permit the subject to extend an arm into the hatch for blood sampling or blood pressure measurement without breaking the air seal between the inside and outside of the calorimeter. The door was held closed by an electromagnetic catch so that in the event of power failure it automatically opened. There was a 'panic button' to enable the subject to open the door from inside the chamber in an emergency.

Air was circulated inside the chamber at about 3000 litres/min by a large fan (F) situated under a bed along the rear wall of the calorimeter. This air emerged from the holes in the perforated hardboard panel on the right, passed in laminar flow to the panel on the left, and thence to the fan, either passing through the main heat exchanger $(X)$ or bypassing it, and so back to the fan again. The proportion of circulating air which passed through 
$\mathrm{X}$ was controlled by a rotating semicircular shutter (S) driven by a servomotor, which responded to changes in heat flow across the walls, monitored by thermistors on the internal and external Al faces of the walls. Thus as the amount of heat generated within the calorimeter changed the shutter turned in the appropriate manner to achieve zero heat flow across the walls.

Two small heat exchangers $(\mathrm{Y}$ and $\mathrm{Z})$ were each supplied with water at $4^{\circ}$ at a flow-rate of $800 \mathrm{ml} / \mathrm{min}$ from a reservoir $(R)$ in the adjacent room: $Y$ cooled fresh air entering the chamber at 40 litres/min and $Z$ cooled an equal amount of air leaving the chamber, so there was no net gain or loss of heat or water vapour in the ventilating system. The water flowing through $Z$ then went to a reference heater (e) which injected exactly 100 watts into the water, and then to the main heat exchanger. The temperature of the water was monitored at points $\mathrm{a}, \mathrm{b}, \mathrm{c}$ and $\mathrm{d}$ by thermistors.

The temperature rise across the reference heater $(c-b)$ represented an input of 100 watts into the stream of water. The temperature rise $(d-a)$ represented the total heat extracted from the calorimeter plus reference heater. Thus, the heat extracted from the calorimeter (E; watts), is given by

$$
E=\frac{(d-a)-(c-b)}{(c-b) \times 100}
$$

Some of this heat was generated by the fan, light, television set and cassette recorder, but this was registered on a wattmeter (W). The heat loss of the subject is $\mathrm{E}-\mathrm{W}$ (watts).

If the heat loss of the subject did not equal heat production then body temperature would not remain constant. We assumed that the thermal mass of the subject was equal to that of an equal weight of water: thus if the oral temperature of the subject changed by $1^{\circ}$ it was assumed that body-weight $(\mathrm{kg}) \times 4.2 \mathrm{~kJ}$ had been gained or lost from the heat store of the body. We determined that the thermal equivalent of the calorimeter chamber is equivalent to $100 \mathrm{~kg}$ water, so if the ambient temperature at the start and finish of an experiment was not identical a similar correction was applied for heat storage in the calorimeter.

The output of the calorimeter was registered on a multichannel recorder which showed the temperature gradient across the walls of the chamber, the rate of heat extraction, the integral of the rate of heat extraction, the ambient temperature, and the heart rate (Weller, 1977) and physical activity of the subject as measured by an ultrasonic detector (Model 8181; C. F. Palmer, London).

Calibration of the calorimeter was achieved with two types of standard. An electrical heat source of known energy dissipation could be switched on from outside the calorimeter, and the known energy input was compared with the heat recovered. Alternatively, to check also the recovery of heat in water vapour, a butane lamp was used as an energy source. The energy equivalent of butane was stated by the manufacturer to be $49 \cdot 233 \mathrm{~kJ} / \mathrm{g}$.

The experimental protocol is illustrated in Fig. 2. The subject wore a light cotton trouser suit with short sleeves. Weight and oral temperature were recorded, electrodes for recording heart rate were put in place, and the subject entered the calorimeter chamber by $09 \cdot 30$ hours. For subjects G.W. and P.P. coffee was provided at $11.00,12.30$ and 15.00 hours and lunch providing $2 \cdot 2 \mathrm{~kJ}$ at 12.30 hours. Subjects J.W. and J. G. had only black coffee.

In the 'relaxed' experimental condition the subject lay on the bed listening to taped music of his or her choice from 09.30 hours to 17.00 hours. The physical activity required to change tapes was similar for the 'relaxed' and 'working' conditions.

In the 'working' condition the subject sat at a table from 09.30 to 17.00 hours. From 10.00 to 12.30 hours, and again from 13.00 to 16.00 hours, he or she played tapes containing items of medical news interspersed with advertizements. Each tape lasted 28-30 min, and the subject was required to enter on a form the time at which each item started, the names 


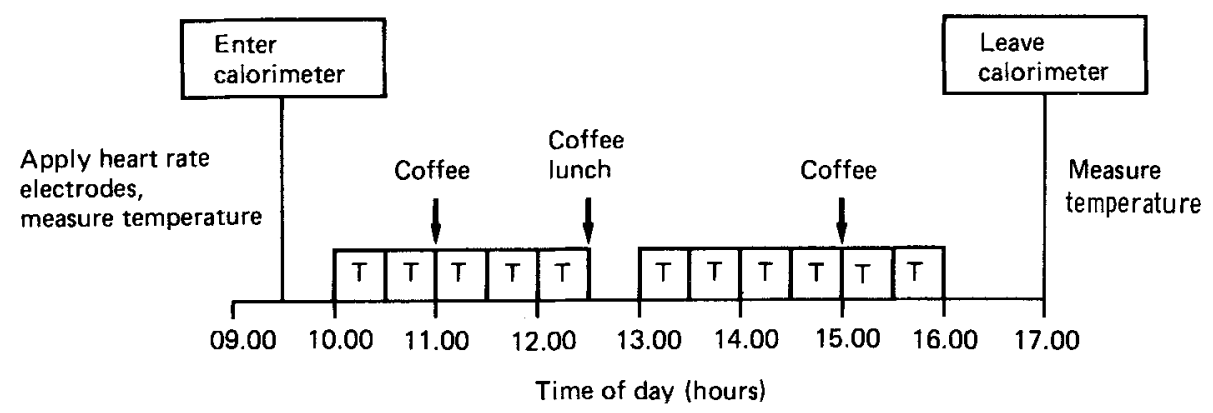

Fig. 2. Diagram of the experimental protocol. At the periods marked $T$ the subject took notes on tape recordings which each lasted 28-30 min on 'working' days, or played music of his or her choice and remained recumbent on 'relaxing' days.

Table 2. Average rate of heat loss (watts) and urinary cortisol excretion over a period of $7.5 \mathrm{~h}$ in four normal subjects either relaxing or doing clerical work

\begin{tabular}{|c|c|c|c|c|c|c|c|c|}
\hline \multirow[b]{2}{*}{ Subject } & \multirow[b]{2}{*}{$\begin{array}{l}\text { Run } \\
\text { no. }\end{array}$} & \multicolumn{3}{|c|}{ Relaxing } & \multicolumn{3}{|c|}{ Working } & \multirow[b]{2}{*}{$\begin{array}{c}\text { Cortisol } \\
(\mathrm{nmol} / 7.5 \mathrm{~h})\end{array}$} \\
\hline & & $\begin{array}{l}\text { Heat } \\
\text { loss }\end{array}$ & Mean & $\operatorname{Var} \%$ & $\begin{array}{l}\text { Heat } \\
\text { loss }\end{array}$ & Mean & $\operatorname{Varn} \%$ & \\
\hline \multirow[t]{5}{*}{ G.W. } & 1 & $70 \cdot 10$ & - & - & - & - & - & $51 \cdot 5$ \\
\hline & 2 & - & - & - & $75 \cdot 20$ & - & - & $32 \cdot 4$ \\
\hline & 3 & - & - & - & $79 \cdot 58$ & - & - & $100 \cdot 9$ \\
\hline & 4 & $70 \cdot 10$ & - & - & - & - & - & $69 \cdot 0$ \\
\hline & & - & $70 \cdot 10$ & $0 \cdot 0$ & - & 77.39 & $5 \cdot 7$ & - \\
\hline \multirow[t]{5}{*}{ J.W. } & 1 & - & - & - & $90 \cdot 86$ & - & - & $110 \cdot 6$ \\
\hline & 2 & $77 \cdot 40$ & - & - & - & - & - & $93 \cdot 3$ \\
\hline & 3 & 67.88 & - & - & - & - & - & $77 \cdot 7$ \\
\hline & 4 & - & - & - & $78 \cdot 68$ & - & - & $76 \cdot 6$ \\
\hline & & - & 72.64 & $13 \cdot 1$ & - & 84.77 & $14 \cdot 4$ & - \\
\hline \multirow[t]{5}{*}{ J.G. } & 1 & - & - & - & $116 \cdot 38$ & - & - & $58 \cdot 2$ \\
\hline & 2 & 110.06 & - & - & - & - & - & 113.6 \\
\hline & 3 & 104.07 & - & - & - & - & - & $149 \cdot 5$ \\
\hline & 4 & - & - & - & 108.57 & - & - & 58.6 \\
\hline & & - & $107 \cdot 7$ & $5 \cdot 6$ & - & $112 \cdot 48$ & 6.9 & - \\
\hline \multirow[t]{5}{*}{ P.P. } & 1 & 62.66 & - & - & - & - & - & $92 \cdot 0$ \\
\hline & 2 & - & - & - & 77.64 & - & - & 113.7 \\
\hline & 3 & - & - & - & $69 \cdot 12$ & - & - & $159 \cdot 8$ \\
\hline & 4 & 70.99 & - & - & - & - & - & 167.2 \\
\hline & & - & $66 \cdot 83$ & 12.5 & - & $73 \cdot 38$ & 11.6 & - \\
\hline Mean & & $79 \cdot 16$ & & & 87.00 & & & - \\
\hline SD & & $17 \cdot 76$ & & & 16.96 & & & - \\
\hline
\end{tabular}

$\mathrm{Vam} \%$, variation between duplicate readings as percentage of mean value.

of any speakers, drugs or journals which were mentioned, and notes on the content of the item. On the replicate day a different set of tapes were supplied.

At 17.00 hours the subject left the calorimeter chamber and weight and oral temperature were again recorded. Excretion of cortisol in urine was measured by the method of Seth \& Brown (1978). Analysis of variance was performed using the Minitab package (Ryan et al. 1981). The protocol was approved by the Northwick Park Hospital Ethical Committee. 


\section{RESULTS}

All the subjects found the 'working' protocol very tiring. The effect of the two protocols on heat loss is shown in Table 2. The mean heat loss for the two working days was greater than that for the two resting days in each subject, the ratio, working: resting heat losses was $1 \cdot 104,1 \cdot 167,1 \cdot 051$ and 1.098 for G. W., J.W., J.G. and P. P. respectively. Analysis of variance showed that the heat loss on the working day was significantly increased $(P=0 \cdot 015)$.

Heart rate, and physical activity as measured by ultrasound, did not differ significantly between the relaxing and working conditions. Urinary cortisol excretion fluctuated widely in each subject (see Table 2) but showed no consistent relation to the working or resting condition.

\section{DISCUSSION}

The average heat loss of the subjects on 'working' days was $10 \%$ greater than on 'relaxing' days. This was a surprisingly small increment in view of the tiring nature of the work task, and even so may have been an overestimate in view of the difference in posture between the two experimental conditions: the 'relaxing' was done lying down while the 'working' was done seated. Warwick (1978) measured the energy expenditure of subjects sitting quietly, and found it to be about $4 \%$ higher than resting metabolism when recumbent, so about $4 \%$ of the $10 \%$ difference may be a posture effect, leaving about $6 \%$ for the energy cost of clerical work.

The absence of any significant effect of the clerical work on heart rate or cortisol excretion is in contrast to the findings in stress induced by mental arithmetic and electric shocks (Blaza \& Garrow, 1980). In that study, heart rate increased from a mean of 75 to 95 beats per min during the $1 \mathrm{~h}$ period of stress, and urinary cortisol excretion increased by about $30 \%$. However, the effect ceased within a few minutes of cessation of the stress, so the effect on mean heart rate and mean cortisol excretion was small over the whole $8 \mathrm{~h}$ period of measurement. The clerical work, although tiring, had no element of anxiety. This probably accounts for the difference in hormonal response to the two types of stress. The cause of the increased energy expenditure is still unclear: it may have been due to increased muscular tension, but we have no direct evidence for this.

The difference in heat loss between duplicate runs in the present study was on average $8.7 \%$ (range $0.0-14.4 \%$ ), which is much inferior to the precision of $24 \mathrm{~h}$ measurements of heat loss by direct calorimetry. This is because, during a period of $7.5 \mathrm{~h}$, there is a change in heat storage in the body while, in $24 \mathrm{~h}$, the body returns to the same point in the circadian cycle and there is relatively little net heat storage. The correction for changes in body temperature which were made in the present study are not satisfactory, since it is certainly an overestimate to assume that the heat stored is equal to the change in core temperature multiplied by body-weight. If no correction for change in heat storage had been made the effect of clerical work would have been estimated to be even smaller, since there was a greater tendency for heat storage during the working days than during the relaxing days.

\section{REFERENCES}

Benedict, F. G. \& Benedict, C. G. (1933) Carnegie Institute Publication no. 446.

Blaza, S. E. \& Garrow, J. S. (1980). Proceedings of the Nutrition Society 39, 13 A.

Blaza, S. E. \& Garrow, J. S. (1983). British Journal of Nutrition 49, 171-180.

Corey, E. L. (1948). Journal of Applied Physiology 1, 35-44.

Landis, C. (1925). American Journal of Physiology 74, 188-203.

Ryan, T. A., Joiner, R. L. \& Ryan, B. F. (1981). Minitab Reference Manual. Pennsylvania: Penn State Press.

Seth, J. \& Brown, L. M. (1978). Clinica Chimica Acta 86, 109-120. 
Tremoliere, J., Carr, L. \& Naon, R. (1973). In Energy Balance in Man, pp. 1256-1134 [M. Apfelbaum, editor]. Paris: Masson.

Udalov, E. F. \& Sibunev, A. G. (1964). Nutrition Abstracts and Reviews 34, 524.

Warwick, P. M. (1978). The influence of physical activity on energy expenditure in man. $\mathrm{PhD}$ thesis, University of London.

Weller, C. (1977). Journal of Physiology 267, 11-12P. 